\title{
Cardioprotective effects of tilianin in rat myocardial ischemia-reperfusion injury
}

\author{
XINHONG GUO $^{1,2^{*}}$, WENJIANG CAO ${ }^{1 *}$, JIAMING YAO $^{1,2}$, YONG YUAN $^{1}$, \\ $\mathrm{YE} \mathrm{HONG}^{1,2}$, XINCHUN WANG ${ }^{1,2}$ and JIANGUO XING ${ }^{3}$ \\ ${ }^{1}$ Department of Pharmacy, First Affiliated Hospital of the Medical College; ${ }^{2}$ School of Pharmacy, Shihezi University, \\ Shihezi, Xinjiang 832000; ${ }^{3}$ Xinjiang Institute of Materia Medica, Ürümqi, Xinjiang 830004, P.R. China
}

Received November 30, 2013; Accepted July 4, 2014

DOI: $10.3892 / \mathrm{mmr} .2014 .2954$

\begin{abstract}
Tilianin, the main effective flavonoid monomer enriched from Dracocephalum moldavuca L., has been shown to have cardioprotective effects. However, the mechanism of tilianin cardioprotection remains largely unknown. The present study aimed to investigate the effects of tilianin preconditioning on myocardial ischemia/reperfusion injury and to analyze the possible mechanism of action. A total of 48 male Sprague Dawley rats were randomized into sham, model myocardial ischemia/reperfusion injury (MI/RI), propranolol hydrochloride positive control, and high-, medium- and low-dose tilianin groups $(n=8$ each). The rats in the tilianin groups were perfused with either $1.5,2.5$ or $5.0 \mathrm{mg} / \mathrm{kg} / \mathrm{d}$ tilianin a week prior to surgery. The positive control group were perfused with $25 \mathrm{mg}$ / $\mathrm{kg} / \mathrm{d}$ propranolol. Saline was administered to the sham surgery and the MI/RI groups. The MI/RI model was established by ligating the left anterior descending coronary artery for $30 \mathrm{~min}$, which was subsequently removed and the mice were observed for 120 min prior to sacrifice. $\mathrm{Na}^{+}-\mathrm{K}^{+}$-ATPase, $\mathrm{Ca}^{2+}$-ATPase, nitric oxide (NO), nitric oxide synthase (NOS) and endothelial system-related factors were analyzed using the respective detection kits. Immunohistochemistry was used to determine the expression levels of Bcl-2 and Bax. Caspase-3 activity was measured by quantitative polymerase chain reaction. The results showed that tilianin preconditioning significantly increased ATPase activity $(\mathrm{P}<0.01$ and $\mathrm{P}<0.05)$ as compared with the model group. With regards to the regulation of endothelial function, significant decreases $(\mathrm{P}<0.01$ and $\mathrm{P}<0.05)$ were detected
\end{abstract}

Correspondence to: Mrs. Xinchun Wang, Department of Pharmacy, First Affiliated Hospital of the Medical College, Shihezi University, 52 Beier Road, Shihezi, Xinjiang 832000, P.R. China E-mail: cwjwxc@163.com

Mr. Jianguo Xing, Xinjiang Institute of Materia Medica, 140 Xinhuanan Road, Ürümqi, Xinjiang 830004, P.R. China E-mail: xingjianguo642@sohu.com

*Contributed equally

Key words: tilianin, myocardial, ischemia/reperfusion, apoptosis in the serum NO levels and myocardial NOS activity when tilianin was administered to MI/RI rats, as compared with the model group, . In addition, the tilianin drug groups exhibited dose-dependent reductions in the serum levels of endothelin 1 and thromboxane B2, and increases in the serum levels of calcitonin gene-related peptide and 6-keto prostaglandin Fla as compared with the model group $(\mathrm{P}<0.01$ and $\mathrm{P}<0.05)$. Notably, the administration of tilianin significantly inhibited apoptosis, as evidenced by an increase in $\mathrm{Bcl}-2$ expression, and reductions in Bax and caspase-3 mRNA expression levels $(\mathrm{P}<0.01$ and $\mathrm{P}<0.05)$. These data indicate that pretreatment with tilianin exerts potent cardioprotective effects in rats with MI/RI. The anti-MI/RI effects comprised relieving calcium overload, correction of energy metabolism, improvement of endothelial function and inhibiting cell apoptosis.

\section{Introduction}

Cardiovascular disease is one of the most common life-threatening diseases, and it has been predicted that it will be the leading cause of mortality worldwide by 2030 (1). The predominant cause of mortality in cardiovascular disease is an acute myocardial infarction (AMI). Restoration of the blood flow to the ischemic myocardium is the most effective treatment principle of AMI. Therapeutic strategies, including thrombolysis and primary angioplasty (2), are frequently used in clinical practice. Nevertheless, reperfusion is considered to pose a risk, as it may result in a worsening of the tissue injury (3) and myocardial ischemia reperfusion injury (MI/RI), which may paradoxically reduce the beneficial effects of myocardial reperfusion, resulting in contractile dysfunction and cellular damage (4). The pathogenesis of MI/RI is complex and the mechanisms involved have not yet been fully elucidated. Previous studies have shown that active oxygen, calcium overload, neutrophil infiltration and apoptosis are all involved in the occurrence of MI/RI (5-9).

Dracocephalum moldavuca $L$. is a herb from the Labiatae family. This herb is traditionally used in Uyghur Medicine for the treatment of coronary heart disease, hypertension, atherosclerosis and myocardial ischemic disease in China (10). The predominant chemical constituents of Dracocephalum moldavuca $L$. are flavonoids, terpenoids, volatile oils, amino acids, trace elements and peptides. Tilianin is a predominant 
effective flavonoid monomer enriched from Dracocephalum moldavuca L. (11). As this traditional Chinese medicine monomer has a clear chemical structure and certain pharmacodynamic advantages, such as improved targeting, the utilization of Chinese medicine resources to analyze the efficacy of this compound is currently a key area of investigation. Nam et al (12) suggested that tilianin inhibits inducible nitric oxide synthase (iNOS) expression and production of nitric oxide (NO), and may act as an anti-inflammatory agent. Another previous study revealed tilianin to exert a protective effect in MI/RI (13), but the protective mechanism remains largely unknown. Therefore, further studies are required regarding the protective effects and mechanisms of tilianin on MI/RI

The present study aimed to continue to investigate the cardioprotective effects of tilianin on MI/RI and elucidate its mechanism of action, in order to provide a theoretical basis for the clinical treatment of coronary heart disease with tilianin.

\section{Materials and methods}

Drugs and reagents. Tilianin was enriched from Dracocephalum moldavica $L$. in the laboratory, and ${ }^{1} \mathrm{H}$-nuclear magnetic resonance (NMR), ${ }^{13} \mathrm{C}$-NMR, mass spectrometry and infrared spectrometry were used to identify the chemical structure. The purity obtained was $>98 \%$, as measured by high-performance liquid chromatography. The propranolol hydrochloride tablet (PHT) was purchased from Tianjin Lisheng pharmaceutical Co., Ltd (Tianjin, China) and sodium pentobarbital was supplied by Beijing Chemical Reagent Company (Beijing, China). The 2X Taq polymerase chain reaction (PCR) MasterMix was bought from Tiangen Biological Technology Co., Ltd (Beijing, China). The $\beta$-actin and caspase-3 primers were obtained from Bioneer Corporation (Daejeon, Korea).

Animals. The animal experiments carried out in the present study were approved by the Institutional Animal Care and Use Committee at Xinjiang Uyghur Autonomous Region Experimental Animal Research Center (Xinjiang, China). A total of 48 male Sprague Dawley rats, weighing 250-300 g, were provided by the Xinjiang Uyghur Autonomous Region Experimental Animal Research Center (certificate no.: XK 2003-0001; Xinjiang, China) and were housed under conditions of constant temperature and controlled illumination (light on between 8:30 and 20:30 h). Food and water were available ad libitum.

In vivo myocardial ischemia and reperfusion model. All animals were assigned randomly to one of four groups: Sham group, rats were pretreated with saline, $n=8 ; M I / R I$ group, rats were pretreated with saline, $\mathrm{n}=8 ; \mathrm{MI} / \mathrm{RI}+$ tilianin groups, high-, medium- and low-dose group rats were pretreated with tilianin $(5.0 \mathrm{mg} / \mathrm{kg}, 2.5 \mathrm{mg} / \mathrm{kg}, 1.5 \mathrm{mg} / \mathrm{kg}$ respectively), $\mathrm{n}=24$, 8 rats in each group; MI/RI + PHT group, rats were pretreated with PHT $(25.0 \mathrm{mg} / \mathrm{kg}), \mathrm{n}=8$. Each treatment was orally administered for eight days, at a volume of $5 \mathrm{ml} / \mathrm{kg}$ weight once a day, and the surgical procedure was established $10 \mathrm{~min}$ after the last administration.
The rats were anesthetized with sodium pentobarbital ( $45 \mathrm{mg} / \mathrm{kg}$ injected intraperitoneally), and then intubated and artificially ventilated using a rodent ventilator (HX-100E; Chongqing Taimeng Technology Co., Ltd., Chongqing, China). A normal electrocardiogram was recorded following the placement of subcutaneous electrodes connected to an electrocardiograph (BL-420S; Chongqing Taimeng Technology Co., Ltd.). Ischemia/reperfusion was established as previously described by Zhao et al (14). Briefly, myocardial ischemia was induced by ligating the left anterior descending coronary artery (LAD) with a $3-0$ silk suture. After $30 \mathrm{~min}$ ischemia, the ligature was released for $2 \mathrm{~h}$, resulting in myocardial reperfusion $(15,16)$. Sham-operated animals (sham group) underwent the same surgical procedures, with the exception that the 3-0 silk was passed around the left coronary artery but not tied. Successful myocardial ischemia was confirmed by ST segment elevation in the electrocardiogram, in addition to visual assessment of regional cyanosis of the ischemic region in the left ventricle. Reperfusion was confirmed by ST segment reversal and a color change in the ventricular surface from cyanotic to hyperemic (16). Arterial blood samples were collected at the end of the reperfusion, and the blood serum was separated at $1,200 \mathrm{x} \mathrm{g}$ and stored at $70^{\circ} \mathrm{C}$. The mouse hearts were removed following the collection of blood and immediately placed in cold saline. The intracardiac blood was rinsed off and the left ventricle tissue was line-clipped under ligation and cryopreserved.

Measurement of $\mathrm{Na}^{+}-\mathrm{K}^{+}$-ATPase and $\mathrm{Ca}^{2+}$-ATPase activity. Briefly, the frozen myocardial tissue was weighted and normal saline was added at a ratio of 1:9. Subsequently, the tissue homogenates were centrifuged to obtain the supernatant, followed by measurement of protein concentration using Coomassie Brilliant Blue kit (Nanjing Jiancheng Bioengineering Institute). $\mathrm{Na}^{+}-\mathrm{K}^{+}$-ATPase and $\mathrm{Ca}^{2+}$-ATPase activity levels were determined by measuring the release of inorganic phosphate from ATP using the $\mathrm{Na}^{+}-\mathrm{K}^{+}$-ATPase kit and the $\mathrm{Ca}^{2+}$-ATPase kit (Nanjing Jiancheng Bioengineering Institute, Nanjing, China), according to the manufacturer's instructions.

Measurement of NO and NOS activity. NO is chemically reactive and and can quickly convert to nitrate $\left(\mathrm{NO}_{3}{ }^{-}\right)$ and nitrite $\left(\mathrm{NO}_{2}^{-}\right)$in vivo, with $\mathrm{NO}_{2}^{-}$further converting to $\mathrm{NO}_{3}^{-}$. In the assay, $\mathrm{NO}_{3}{ }^{-}$was reduced to $\mathrm{NO}_{2}{ }^{-}$using nitrate reductase. The absorbance of $\mathrm{NO}_{2}{ }^{-}$at $550 \mathrm{~nm}$ indirectly signifies the NO concentrations. NOS catalyzes L-Arg and molecular oxygen to produce NO and pronuclear material, which generates colored compounds. Thus, the absorbance at the $530 \mathrm{~nm}$ wavelength allows for calculation of the NOS activity. Using the serum and homogenate supernatant of each group, the NO levels and NOS activity were determined with a power wave XS2 enzyme-labeled instrument (Bio-Rad Laboratories, Hercules, CA, USA) using NO and NOS kits according to the manufacturer's instructions (Nanjing Jiancheng Bioengineering Institute).

Measurement of endothelial system-related factors. The serum levels of endothelin (ET)-1, calcitonin gene-related 
peptide (CGRP), thromboxane (TX) $\mathrm{B}_{2}$ and 6-keto prostaglandin (PG) F1a (6-Keto- GFF $_{1 \mathrm{a}}$ ) in the rat blood samples were measured using the corresponding rat ELISA kits (Shanghai Xitang Biological Technology Co., Ltd., Shanghai, China).

Immunohistochemistry. A section of ischemic tissue was fixed in $4 \%$ paraformaldehyde solution. After $24 \mathrm{~h}$, this was dehydrated and then embedded in paraffin blocks. The blocks were then cut into $5 \mu \mathrm{m}$ sections using a rotary microtome (Leica RM 2235; Leica Instrument Co., Ltd., Beijing, China).

Immunohistochemical procedures were conducted according to the manufacturer's instructions of the streptavidin-peroxidase kit (ZSGB-BIO, Beijing, China). Briefly, the tissue sections were dewaxed and dehydrated with xylene and alcohol. The sections were then incubated for 10 min with $3 \%$ $\mathrm{H}_{2} \mathrm{O}_{2}$ and an autoclave was used to heat the samples for $8 \mathrm{~min}$ for antigen retrieval. The samples were then incubated with the following primary antibodies: Bax (B-9; dilution 1:25; Santa Cruz Biotechnology, Inc., Santa Cruz, CA, USA) and Bcl-2 (C-2; dilution 1:50; Santa Cruz Biotechnology, Inc.) overnight at $4^{\circ} \mathrm{C}$. Following three washes with phosphate-buffered saline, the sections were incubated with a non-biotin labeled goat anti-mouse IgG secondary antibody (ZSGB-BIO) for $30 \mathrm{~min}$ and further developed with 3,3-diaminobenzidine tetrahydrochloride (DAB). The sections were observed under an Olympus CX21 microscope (Xiamen Sannuoxinu Electronic Technology Co., Ltd., Xiamen, China). The immunopositive cell rate was calculated as follows: Immunopositive cell rate $=$ immunopositive cells/(immunopositive cells + immunonegative cells) x100\%. The expression levels of the analyzed proteins were statistically compared using the staining intensity plus the percentage of positive cells. The score criteria are listed in Table I (17).

Semi-quantitative (q) PCR analysis. A centrifugal columnar RNAprep Pure Tissue kit (Tiangen Biological Technology Co., Ltd., Beijing, China) was used to extract the total RNA from the myocardial tissue. cDNA was first synthesized by reverse transcription PCR. An appropriate quantity of total RNA was used to synthesize cDNA using a reverse transcriptase kit (Thermo Fisher Scientific, Rockford, IL, USA) at the following conditions: $65^{\circ} \mathrm{C}$ for $5 \mathrm{~min}, 42^{\circ} \mathrm{C}$ for $60 \mathrm{~min}$ and $70^{\circ} \mathrm{C}$ for $5 \mathrm{~min}$. Subsequently, this cDNA served as a template for the subsequent qPCR reaction: $94^{\circ} \mathrm{C}$ for $3 \mathrm{~min}$, $94^{\circ} \mathrm{C}$ for $30 \mathrm{sec}, 53^{\circ} \mathrm{C}$ for $30 \mathrm{sec}, 72^{\circ} \mathrm{C}$ for $1 \mathrm{~min}$ and $72^{\circ} \mathrm{C}$ for $5 \mathrm{~min} ; 35$ cycles. The following primers were used in the reaction: $\beta$-actin forward, 5 '-AGCCATGTAC GTAGCCATCC-3' and reverse, 5'-CTCTCAGCTGTGGTGAA-3'; caspase-3 forward, 5'-TTGGAGCACTGTAGCACACA-3' and reverse, 5'-ACCACTGAAGGATGGTAGCC-3'. The PCR products were detected by ionization on a $1.5 \%$ agarose gel.

Statistical analysis. Data are expressed as the means \pm standard deviation. A Student's t-test was performed to analyze the differences between two groups. Data analyses were performed using the SPSS 17.0 software package (SPSS, Inc., Chicago, IL, USA). A $\mathrm{P}<0.05$ was considered to indicate a statistically significant difference.
Table I. Immunohistochemical staining criteria.

\begin{tabular}{lccc}
\hline $\begin{array}{l}\text { Staining } \\
\text { intensity }\end{array}$ & Score & $\begin{array}{c}\text { Immunopositive } \\
\text { cell rate (\%) }\end{array}$ & Score \\
\hline Colorless & 0 & 0 & 0 \\
Buff & 1 & $\leq 25$ & 1 \\
Yellow & 2 & $26-50$ & 2 \\
Brown & 3 & $51-75$ & 3 \\
N/A & N/A & $\geq 75$ & 4 \\
\hline
\end{tabular}

A

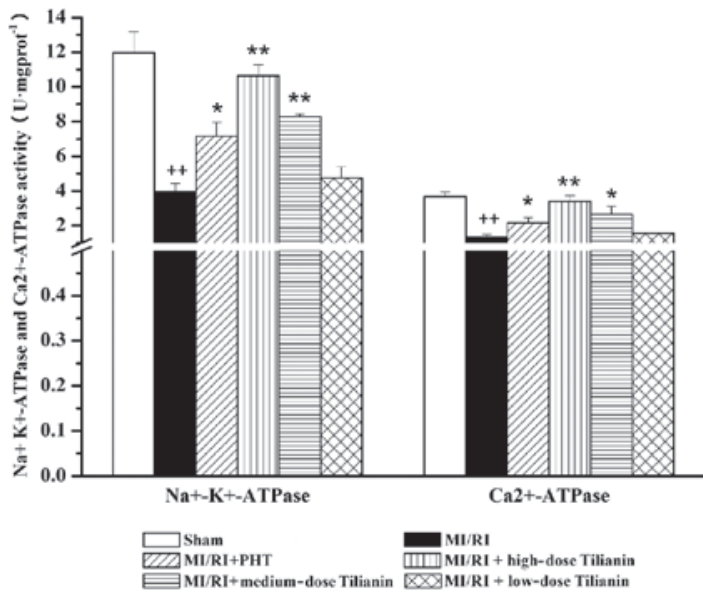

B

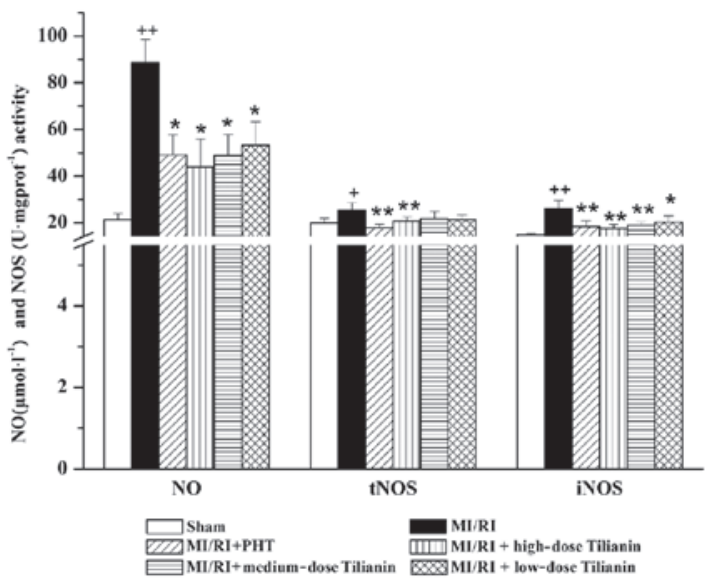

Figure 1. Effect of Tilianin on energy metabolism enzymes and NO activity in rat cardiac tissues. ATPase activity, $\mathrm{NO}$ level and myocardial NOS activity were measured. (A) Effect on $\mathrm{Na}^{+}-\mathrm{K}^{+}$-ATPase and $\mathrm{Ca}^{2+}$-ATPase activities following $\mathrm{MI} / \mathrm{RI}$ in each group. (B) Effect on $\mathrm{NO}$ level and myocardial NOS activity following MI/RI in each group. ${ }^{++} \mathrm{P}<0.01,{ }^{+} \mathrm{P}<0.05$ vs. Sham, ${ }^{*} \mathrm{P}<0.01,{ }^{*} \mathrm{P}<0.05$ vs. MI/RI. NO, nitric oxide; MI/RI, myocardial ischemia/reperfusion injury; NOS, NO synthase; PHT, propranolol hydrochloride tablet; tNOS, total NOS; iNOS, inducible NOS.

\section{Results}

Effect of tilianin on $\mathrm{Na}^{+}-\mathrm{K}^{+}$-ATPase and $\mathrm{Ca}^{2+}$-ATPase activity. The activity of $\mathrm{Na}^{+}-\mathrm{K}^{+}$- and $\mathrm{Ca}^{2+}$-ATPases in myocardial tissues was investigated in response to MI/RI. As compared with the sham group, $\mathrm{Na}^{+}-\mathrm{K}^{+}$- and $\mathrm{Ca}^{2+}$-ATPase activity was significantly reduced in the MI/RI group $\left(\mathrm{Na}^{+}-\mathrm{K}^{+}\right.$-ATPase: sham, 11.97 \pm 1.21 versus MI/RI, 3.96 $\pm 0.48, \mathrm{P}<0.01 ; \mathrm{Ca}^{2+}$-ATPase: sham, $3.66 \pm 0.28$ versus $\mathrm{MI} / \mathrm{RI}, 1.34 \pm 0.15, \mathrm{P}<0.01)$. As 
compared with the MI/RI group, significant recoveries in $\mathrm{Na}^{+}-\mathrm{K}^{+}$-ATPase and $\mathrm{Ca}^{2+}$-ATPase activities were observed in the high- and medium-dose tilianin groups $(\mathrm{P}<0.01$ and $\mathrm{P}<0.05$ respectively), whereas no significant differences were observed between the low-dose tilianin group and the MI/RI group. The MI/RI + PHT group also exhibited a statistically significant increase in ATPase activity, in comparison with the $\mathrm{MI} / \mathrm{RI}$ group $(\mathrm{P}<0.05$; Fig. $1 \mathrm{~A})$.

Effect of tilianin on NO and NOS activity. As shown in Fig. 1B, serum NO levels and myocardial NOS activity were significantly increased following MI/RI surgery, as compared with the sham group [NO: sham, 21.38 2.66 versus MI/RI, $88.49 \pm 10.11, \mathrm{P}<0.01$; Total $(\mathrm{t}) \mathrm{NOS}$ : sham, $19.87 \pm 2.08$ versus $\mathrm{MI} / \mathrm{RI}, 25.40 \pm 3.24, \mathrm{P}<0.05$; iNOS: sham, $14.88 \pm 0.51$ versus $\mathrm{MI} / \mathrm{RI}, 26.04 \pm 3.51, \mathrm{P}<0.01]$. In comparison with the MI/RI group, significant decreases $(\mathrm{P}<0.01$ and $\mathrm{P}<0.05)$ were identified in the serum NO levels and myocardial iNOS activity upon administration of tilianin to MI/RI rats at the three dose levels analyzed. Only the high-dose tilianin group significantly reduced tNOS activity, as compared with the MI/RI group $(\mathrm{P}<0.01)$

Effect of tilianin on endothelial system-related factors. The tilianin drug groups exhibited dose-dependent reductions in the levels of serum ET-1 and $\mathrm{TXB}_{2}$, and increases in the levels of serum CGRP and 6-Keto-PGF ${ }_{1 a}$, as compared with the MI/RI group. The high- and medium-dose groups significantly differed from the model group (ET-1: MI/RI, 34.63 \pm 4.32 vs. MI/RI + high-dose tilianin, 21.64 $\pm 2.82, \mathrm{P}<0.01$; CGRP: MI/RI, 1,479.58 \pm 235.29 vs. MI/RI + high-dose tilianin, 2,036.06 $\pm 261.24, \mathrm{P}<0.01 ; \mathrm{TXB}_{2}: \mathrm{MI} / \mathrm{RI}, 2,480.05 \pm 317.55$ versus $\mathrm{MI} / \mathrm{RI}+$ high-dose tilianin, $1,717.45 \pm 241.44, \mathrm{P}<0.01$; 6-Keto-PGF ${ }_{1 \mathrm{a}}$ : MI/RI, 30.13 \pm 4.72 versus $\mathrm{MI} / \mathrm{RI}+$ high-dose tilianin, 79.56 $\pm 7.90, \mathrm{P}<0.01)$. However, the MI/RI + PHT group exhibited reduced ET-1 and $\mathrm{TXB}_{2}$ levels and increased CGRP and 6-Keto-PGF la $_{1}$ levels as compared with all the tilianin-treated groups. (Fig. 2)

Effect of tilianin on Bcl-2 and Bax expression levels. Bcl-2 protein was mainly accumulated in the cytoplasm. Immunohistochemical DAB coloration revealed diffuse distribution or brown granules in the cell cytoplasm. In the sham group, a few particles of Bcl-2 protein were detected (Fig. 3A). In the model group, positive staining of $\mathrm{Bcl}-2$ protein was significantly increased in the cytoplasm $(\mathrm{P}<0.05)$. As compared with the model group, the tilianin drug groups exhibited significantly increased Bcl-2 protein expression levels $(\mathrm{P}<0.01$ and $\mathrm{P}<0.05$ for the high- and medium-dose groups, respectively; Fig. 3B).

The expression levels of Bax protein were similar to those of Bcl-2 protein, with an accumulation of Bax observed in the cytoplasm. Immunohistochemical staining revealed the sham group to exhibit almost no Bax protein expression (Fig. 3C); however, the Bax protein expression levels in the model group were significantly increased $(\mathrm{P}<0.01$; Fig. 3D), as compared with those of the sham group These data indicate that myocardial damage promotes apoptotic protein expression. Compared with the model group, the high- and medium-dose tilianin drug groups exhibited significantly decreased Bax protein expression levels $(\mathrm{P}<0.01)$. In addition, the high- and
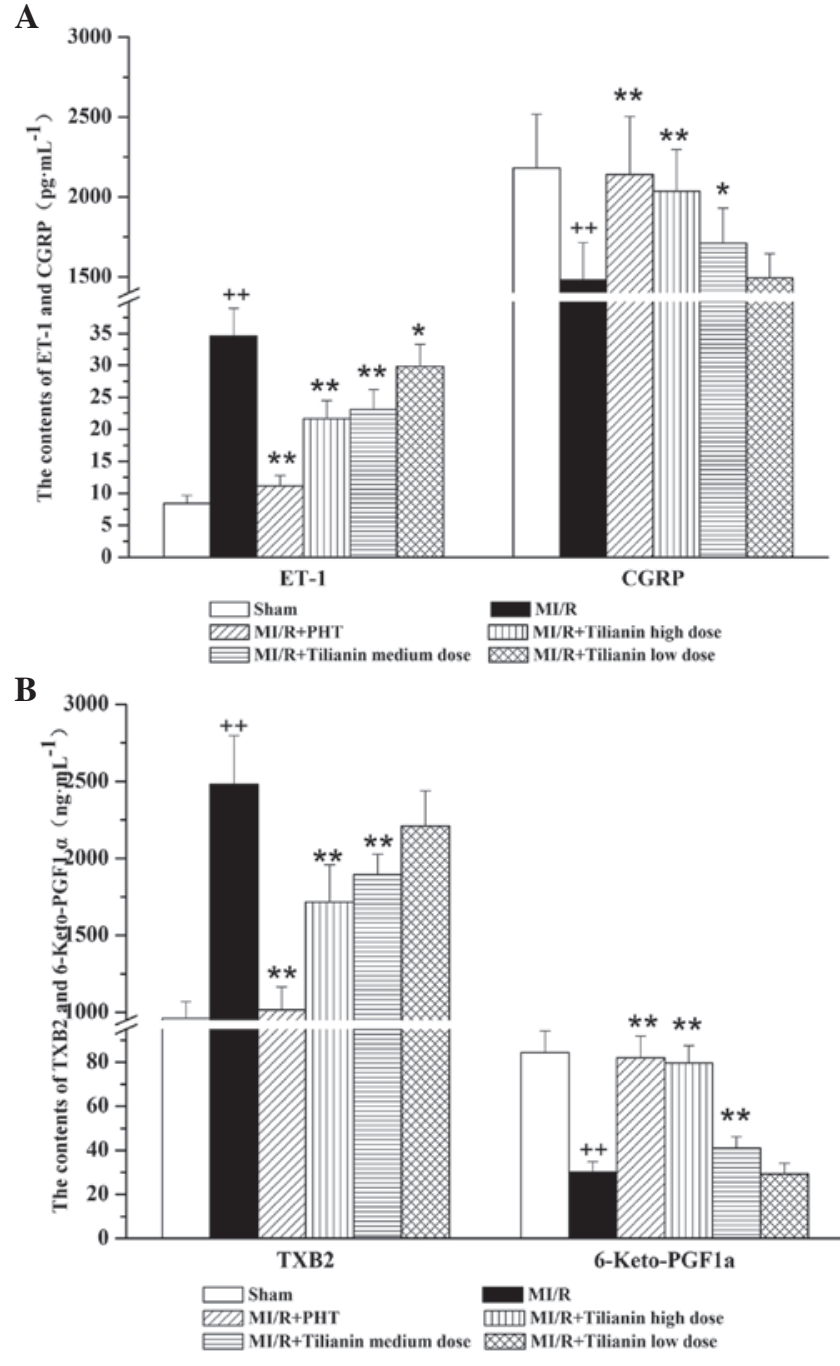

Figure 2. Effect of Tilianin on endothelial system-related factors, measured by ELISA. (A) Serum levels of ET-1 and CGRP in each group. (B) Serum level of $\mathrm{TXB}_{2}$ and 6-Keto- $\mathrm{PGF}_{1 \mathrm{a}}$ in each group. ${ }^{++} \mathrm{P}<0.01$ vs. Sham, ${ }^{* *} \mathrm{P}<0.01$, ${ }^{*} \mathrm{P}<0.05$ vs. MI/RI. ET-1, endothelin 1; CGRP, calcitonin gene-related peptide, $\mathrm{TXB}_{2}$, thromboxane B2; 6-Keto-PGF $1 \mathrm{a}$, 6-keto prostaglandin F1a; $\mathrm{MI} / \mathrm{RI}$, myocardial ischemia/reperfusion injury; PHT, propranolol hydrochloride tablet.

medium-dose tilianin drug groups exhibited significantly increased $\mathrm{Bcl}-2 / \mathrm{Bax}$ ratio, as compared with the model group $(\mathrm{P}<0.01$; Fig. 3E).

Effect of tilianin on caspase-3 mRNA expression levels. Compared with the sham group, the expression levels of caspase-3 mRNA in the MI/RI group were significantly increased (sham, 0.37 \pm 0.037 versus MI/RI, 1.04 \pm 0.07 , $\mathrm{P}<0.01)$. As compared with the MI/RI group, the tilianin highand medium-dose groups exhibited significantly reduced caspase-3 mRNA expression levels (high-dose tilianin, $0.56 \pm 0.09, \mathrm{P}<0.01$; medium-dose tilianin, 0.88 $\pm 0.06, \mathrm{P}<0.05$ ). In addition, he MI/RI + PHT group exhibited decreased expression levels of caspase-3 mRNA, as compared with those of the MI/RI group (MI/RI + PHT, $0.42 \pm 0.03, \mathrm{P}<0.01)$. The results indicated that tilianin exerted a protective effect on the cardiovascular tissue that underwent MI/RI, by reducing the expression levels of caspase-3 mRNA (Fig. 4). 
A

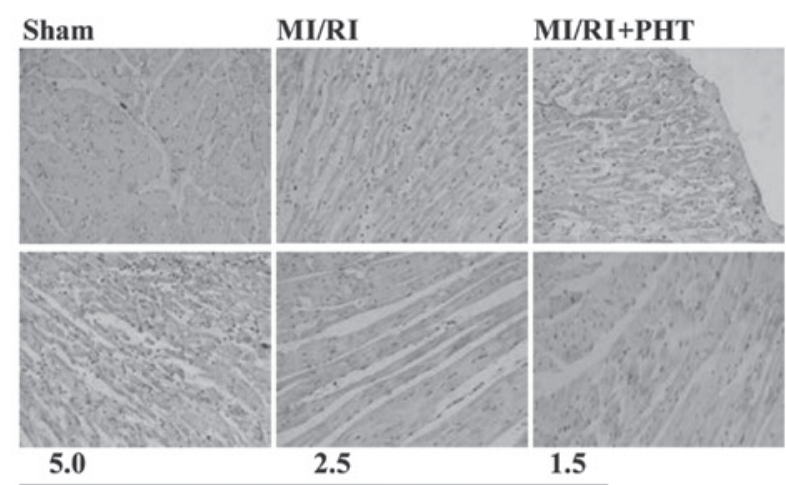

MI/RI + Tilianin (mg/kg)

C

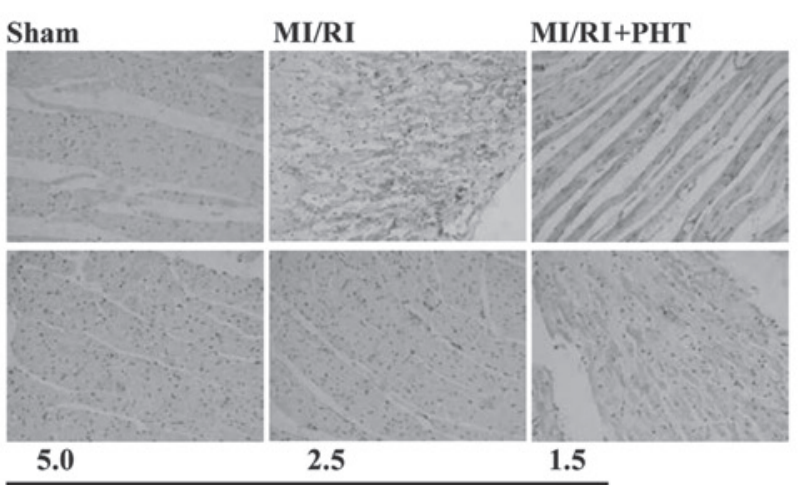

MI/RI + Tilianin (mg/kg)
B

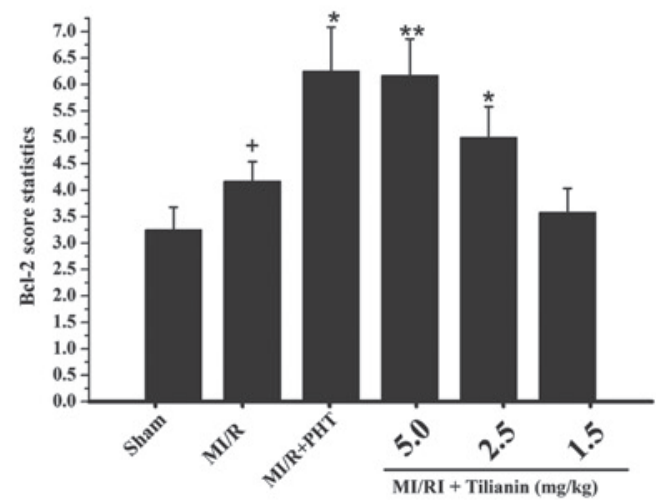

D

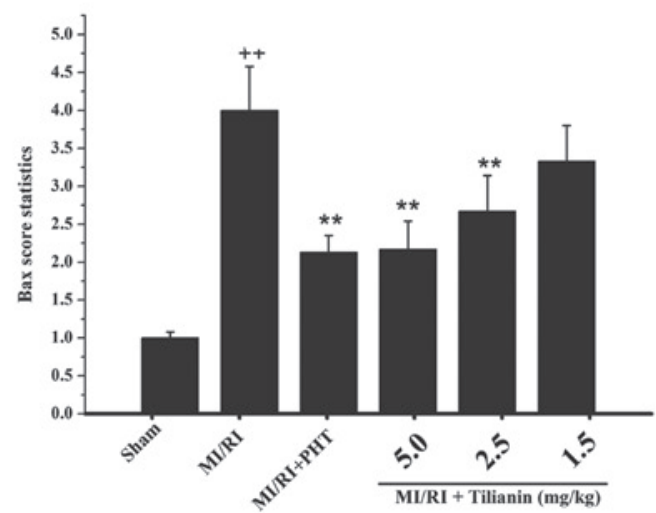

E

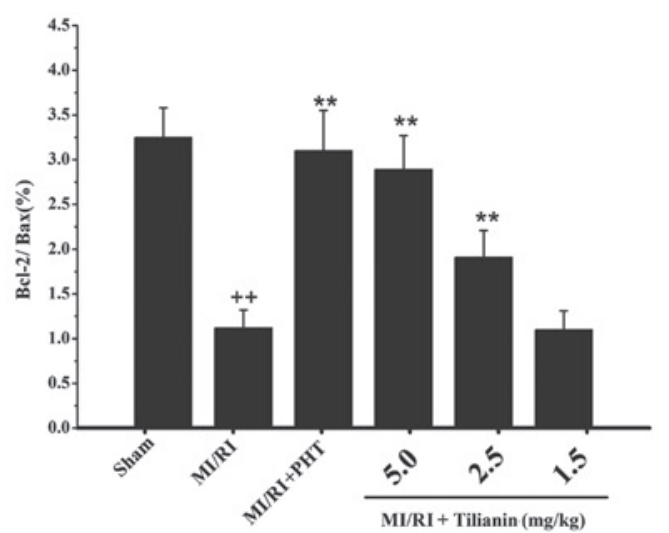

Figure 3. Bcl-2 and Bax expression levels following MI/RI in each group of rats. (A) Expression levels of Bcl-2 protein in each group (magnification, x200), (B) Statistical analysis of Bcl-2 protein expression. (C) Expression levels of Bax protein in each group (magnification, x200). (D) Statistical analysis of Bax protein expression. (E) Statistical analysis of of Bcl-2/Bax (\%). ${ }^{++} \mathrm{P}<0.01,{ }^{+} \mathrm{P}<0.05 \mathrm{vs}$. Sham; ${ }^{* *} \mathrm{P}<0.01,{ }^{*} \mathrm{P}<0.05 \mathrm{vs}$. MI/RI. MI/RI, myocardial ischemia/reperfusion injury; PHT, propranolol hydrochloride tablet.

A

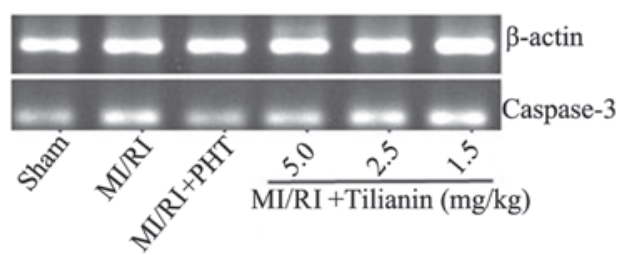

B

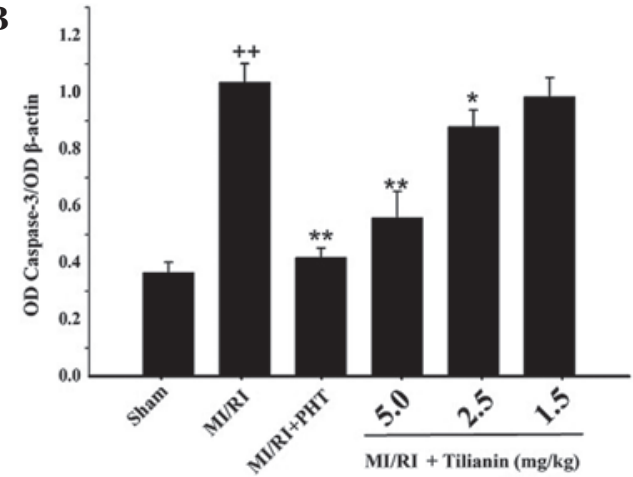

Figure 4. Caspase-3 mRNA expression levels in the myocardial tissue of each group. (A) Caspase-3 mRNA expression levels in each group. (B) Caspase-3/ $\beta$-actin OD statistics. ${ }^{++} \mathrm{P}<0.01$ vs. Sham, ${ }^{* *} \mathrm{P}<0.01,{ }^{*} \mathrm{P}<0.05$ vs. MI/RI. OD, optical density; MI/RI, myocardial ischemia/reperfusion injury; PHT, propranolol hydrochloride tablet. 


\section{Discussion}

The present study generated three notable findings: (i) Pretreatment with tilianin attenuated calcium overload and energy metabolism disorders in MI/RI, a condition that predominantly manifests through increased $\mathrm{Na}^{+}-\mathrm{K}^{+}$-ATPase and $\mathrm{Ca}^{2+}$-ATPase activity; (ii) reduced endothelial system-related factors, including NO and endothelial NOS, significantly contributed to the cardioprotective effect of tilianin; (iii) tilianin was demonstrated to protect against MI/RI by inhibiting apoptosis.

Traditional Chinese herbs are used worldwide and analyses of the effective components are being widely investigated. A number of studies $(18,19)$ have confirmed that Dracocephalum moldavuca $L$. total flavonoid exerts a protective effect on MI/RI. Tilianin is an effective ingredient extracted from Dracocephalum moldavuca $L$. Previous studies have confirmed that the cardioprotective effects of tilianin in MI/RI are associated with reduced levels of intracellular enzymes that are released into the blood, attenuation of the overproduction of reactive oxygen species and reductions in the release of inflammatory factors, such as IL-1, IL-6 and tumor necrosis factor- $\alpha$ (13).

$\mathrm{Na}^{+}-\mathrm{K}^{+}$-ATPase is an integral membrane protein that maintains the normal physiological gradient across the cell membrane. This is achieved through coupling ATP hydrolysis to the transport of $\mathrm{Na}^{+}$and $\mathrm{K}^{+}$. The $\mathrm{Na}^{+}-\mathrm{K}^{+}$-ATPase enzyme is comprised of two subunits, both of which have numerous isoforms and ion-pumping functions (20). For the transport of calcium ions, $\mathrm{Ca}^{2+}$-ATPase is the predominant active transport protein that can regulate intracellular calcium levels in numerous cell types. The $\mathrm{Ca}^{2+}$-ATPase has an important role in maintaining the cation gradient for homeostatic control of cellular properties, and is also crucial for the contractility and excitability of muscles $(21,22)$. In the present study, $\mathrm{Na}^{+}-\mathrm{K}^{+}$-ATPase and $\mathrm{Ca}^{2+}$-ATPase activity was found to be reduced in the MI/RI group, but a significant recovery was observed in the tilianin drug group (Fig. 1). The results suggested that tilianin may protect the myocardium by increasing ATP enzyme activity, maintaining homeostasis of the intracellular environment and rectifing energy metabolism.

A number of studies have confirmed the cardioprotective effects of NO during MI/RI. In addition, the effects of $\mathrm{NO}$ on MI/RI that have been demonstrated include pro- and antiapoptotic effects, depending on the source of NO $(23,24)$. However, the mechanisms underlying these effects are not completely understood. Previous studies have indicated that the beneficial effects of NO from NOS are mediated by the regulation of vascular tone, superoxide radical scavenging, and the inhibition of neutrophil adherence and infiltration $(9,25)$. The results of the present study were contrary to those of previous studies, showing that myocardial NO expression levels and NOS activity was markedly increased following MI/RI injury. However, the rats of the tilianin drug-treatment groups exhibited significantly lower levels of NO and NOS (Fig. 1). These data indicate that tilianin may protect against $\mathrm{MI} / \mathrm{RI}$, by reducing myocardial NOS activity and decreasing the production of $\mathrm{NO}$, therefore reducing the cytotoxic effects of NO.
The endothelial system-related factors ET-1 and CGRP were investigated following tilianin administration. ET-1 is one of the most potent endogenous vasoconstrictor peptides so far identified (26); CGRP is a vasoactive peptide that exhibits a variety of physiological functions, including vasodilation (27). Together, ET-1 and CGRP are a pair of vasoconstriction and vasodilation factors that are mainly regulated through endothelial function. In addition, the dynamic balance of $\mathrm{TXA}_{2} / \mathrm{PGI}_{2}$ is the main regulatory system in maintaining angiectasis and normal platelet function. However, these molecules are unstable in vivo due to a short half-life. Therefore, the concentrations of $\mathrm{TXA}_{2}$ and $\mathrm{PGI}_{2}$ are determined by monitoring the levels of the respective hydrolysis products, $\mathrm{TXB}_{2}$ and $6-$ Keto- $\mathrm{PGF}_{1 \mathrm{a}}$. In the tilianin drug groups, reduced serum ET-1 and $\mathrm{TXB}_{2}$ levels, and increased serum CGRP and 6-Keto-PGF la $_{\text {a }}$ levels were observed, as compared with the the MI/RI group. The results suggest that tilianin further protects against MI/RI by maintaining the balance of endothelial function-related factors.

Cardiomyocyte apoptosis is a predominant pathogenic mechanism underlying MI/RI injury (28). Apoptosis following ischaemia-reperfusion (I/R) has been determined to be associated with increased levels of Bax protein and a decreased Bcl-2/Bax ratio (29). The overexpression of Bcl-2 in mice has been shown to significantly inhibit apoptosis and decrease the infarct size in the mouse heart following I/R (30). Bax and $\mathrm{Bcl} 2$ are important mitochondrial regulators during cardiomyocyte apoptosis (31). Bcl-2 regulates the opening of mPTP in opposition to Bax, blocking cytochrome c release, inhibiting caspase activity and reducing cell apoptosis (32). Therefore, the roles of Bcl-2 and Bax proteins in the antiapoptotic effects of tilianin were investigated in the present study. The results showed that tilianin preconditioning significantly increased Bcl-2 expression levels and reduced Bax levels, with a corresponding increase in the $\mathrm{Bcl}-2 / \mathrm{Bax}$ ratio. The results indicated that changes in the ratio of proapoptotic to antiapoptotic proteins may also contribute to the antiapoptotic and cardioprotective effects of tilianin in I/R injury (Fig. 3).

Caspases are critical enzymes in the induction and execution of apoptosis, and can induce cellular destruction through the formation of apoptotic bodies. The caspase family has numerous members, and the effector caspase, caspase- 3 , is associated with the apoptotic cascade pathway (33), and is therefore widely used as an indicator of apoptosis. The results of the present study revealed that tilianin exhibited cardioprotective effects by inhibiting caspase-3 activity, and thus the activation of the cleavage process (Fig. 4).

In conclusion, these results demonstrate that tilianin exerts potent cardioprotective effects in rats with MI/RI. The effects of anti-MI/RI observed, included relief of calcium overload, correction of energy metabolism, improvement in endothelial function and inhibition of cell apoptosis. This suggests that tilianin is a potentially useful drug that may be applied clinically for the prevention or treatment of MI/RI.

\section{Acknowledgements}

This study was supported by grants from the National Natural Science Foundation of China (nos. 81160525 and 81360671) and the Xinjiang Production and Construction Corps Applied Basic Research Plan for Doctor (no. 2013BB014). 


\section{References}

1. Kreatsoulas $\mathrm{C}$ and Anand SS: The impact of social determinants on cardiovascular disease. Can J Cardiol 26: 8C-13C, 2010.

2. Hausenloy DJ and Yellon DM: New directions for protecting the heart against ischemia-reperfusion injury: targeting the Reperfusion injury Salvage Kinase (RISK)-pathway. Cardiovasc Res 61: 448-460, 2004.

3. Ambrosio G and Tritto I: Reperfusion injury: experimental evidence and clinical implications. Am Heart J 138: S69-S75, 1999.

4. Yellon DM and Baxter GF: Protecting the ischemic and reperfused myocardium in acute myocardial infarction: distant dream or near reality? Heart 83: 381-387, 2000.

5. Li J,Zhang $\mathrm{H}$ and Zhang C: Role of inflammation in the regulation of coronary blood flow in ischemia and reperfusion: mechanisms and therapeutic implications. J Mol Cell Cardiol 52: 865-872, 2012

6. Gottlieb RA: Cell death pathways in acute ischemia/reperfusion injury. J Cardiovasc Pharmacol Ther 16: 233-238, 2011.

7. Koenitze JR and Freeman BA: Redox signaling in inflammation: interactions of endogenous electrophiles and mitochondria in cardiovascular disease. Ann NY Acad Sci 12: 45-52, 2010.

8. Lakshmi SV, Padmaja G, Kuppusamy P and Kutala VK: Oxidative stress in cardiovascular disease. Indian J Biochem Biophys 46: 421-440, 2009.

9. Liou SF, Ke HJ, Hsu JH, et al: San-Huang-Xie-Xin Tang prevents rat hearts from ischemia/reperfusion-induced apoptosis through eNOS and MAPK pathways. Evid Based Complement Alternat 2011: 915051, 2011.

10. Changgen Feng and Qiong Li: The research of Dracocephalum moldavuca $L$. chemical constituents and pharmacological activities. J Tradit Chin Med 25: 155, 2003.

11. Yong Yuan, JianGuo Xing, YongJun Zhang, et al: Determination the content of tilianin in Dracocephalum moldavuca L. by HPLC. Chin J Exp Tradit Med Formulae 16: 68-69, 2010.

12. Nam KH, Choi JH, Seo YJ, et al: Inhibitory effects of tilianin on the expression of inducible nitric oxide synthase in low density lipoprotein receptor deficiency mice. Exp Mol Med 38: 445-452, 2006

13. Xinhong Guo, Wenjiang Cao, Xinmei Fan, et al: The protective effects of Tilianin on myocardial ischemia-reperfusion injury in rats. Chin J Exp Tradit Med Formulae 13: 169-172, 2013

14. Zhao Q, Shao L, Hu X, Wu G, et al: Lipoxin $\mathrm{A}_{4}$ preconditioning and postconditioning protect myocardial ischemia/reperfusion injury in rats. Mediators Inflamm 2013: 231351, 2013.

15. Wei Q, Yin Y, Xi M, et al: Antioxidant properties of magnesium lithospermate $\mathrm{B}$ contribute to the cardioprotection against myocardial ischemia/reperfusion injury in vivo and in vitro. J Tradit Chin Med 33: 85-91, 2013.

16. Ahmed LA, Salem HA, Attia AS and Agha AM: Pharmacologica preconditioning with nicorandil and pioglitazone attenuates myocardial ischemia/reperfusion injury in rats. Eur J Pharmacol 663: 51-58, 2011.

17. Sun C, Liu C, Li S, et al: Overexpression of GEFT, a Rho family guanine nucleotide exchange factor, predicts poor prognosis in patients with rhabdomyosarcoma. Int J Clin Exp Pathol 7: $1606-1615,2014$

18. Zhao J, Xue W and Liang S: Protective effects of dracocephalum moldavica extracts on hypoxia/reoxygenation injury of cardiomyocytes in cultured neonatal rat. J Zhenzhou University (Med Sci) 2010: 485-487, 2010
19. Fan XM, Cao WJ, Xing JG, et al: Study on the protective effect of Dracocephalum total flavones against myocardial ischemia-reperfusion injury in rats. Chin Tradit Patent Med 8: 1625-1629, 2013 (In Chinese).

20. Rajadurai M and Stanely Mainzen Prince P: Preventive effect of naringin on cardiac markers, electrocardiographic patterns and lysosomal hydrolases in normal and isoproterenol-induced myocardial infarction in Wistar rats. Toxicol 230: 178-188, 2007.

21. Tian D, Dmitrieva RI, Doris PA, et al: Protein kinase M zeta regulation of $\mathrm{Na} / \mathrm{K}$ ATPase: a persistent neuroprotective mechanism of ischemic preconditioning in hippocampal slice cultures. Brain Res 1213: 127-139, 2008.

22. Yorozuya T, Adachi N, Dote $\mathrm{K}$, et al: Enhancement of $\mathrm{Na}^{+} \mathrm{K}^{+}$-ATPase and $\mathrm{Ca}^{2+}$-ATPase activities in multi-cycle ischemic preconditioning in rabbit hearts. Eur J Cardiothorac Surg 26: 981-987, 2004.

23. Yang G, Fang Z, Liu Y, et al: Protective effects of Chinese traditional medicine Buyang Huanwu decoction on myocardial injury. Evid Based Complement Alternat Med 2011: 930324 , 2011.

24. Razavi HM, Hamilton JA and Feng Q: Modulation of apoptosis by nitric oxide: implications in myocardial ischemia and heart failure. Pharmacol Ther 106: 147-162, 2005.

25. Liu YN, Zhou ZM and Chen P: Evidence that hydroxysafflor yellow A protects the heart against ischaemia-reperfusion injury by inhibiting mitochondrial permeability transition pore opening. Clin Exp Pharmacol Physiol 35: 211-216, 2008.

26. Benigni A, Perico N and Remuzzi G: Endothelin antagonists and renal protection. J Cardiovasc Pharmacol 35 (4 Suppl 2): S75-S78, 2000

27. de Hoon JN, Pickkers P, Smits P, et al: Calcitonin gene-related peptide: exploring its vasodilating mechanism of action in humans. Clin Pharmacol Ther 73: 312-321, 2003.

28. Ji L, Fu F, Zhang L, Liu W, Cai X, et al: Insulin attenuates myocardial ischemia/reperfusion injury via reducing oxidative/nitrative stress. Am J Physiol Endocrinol Metab 298: E871-E880, 2010.

29. Moudgil R, Menon V, Xu Y, et al: Postischemic apoptosis and functional recovery after angiotensin II type 1 receptor blockade inisolated working rat hearts. J Hypertens 19: 1121-1129, 2001.

30. Chen Z, Chua CC, Ho YS, et al: Overexpression of Bcl-2 attenuates apoptosis and protects against myocardial I/R injury in transgenic mice. Am J Physiol Heart Circ Physiol 280: H2313-H2320, 2001.

31. Kumar D and Jugdutt BI: Apoptosis and oxidants in the heart. J Lab Clin Med 142: 288-297, 2003.

32. Nishihara M, Miura T, Miki T, et al: Modulation of the mitochondrial permeability transition pore complex in GSK-3beta-mediated myocardial protection. J Mol Cell Cordial 43: 564-570, 2007.

33. Zhang Q, Xiang J, Wang X, et al: Beta(2)-adrenoceptor agonist clenbuterol reduces infarct size and myocardial apoptosis after myocardial ischaemia/reperfusion in anaesthetized rats. Br J Pharmacol 160: 1561-1572, 2010. 